\title{
Supersymmetry in models with strong on-site Coulomb repulsion: Application to the Heisenberg model
}

\author{
T. K. Ng and C. H. Cheng \\ Department of Physics, Hong Kong University of Science and Technology, Kowloon, Hong Kong, China
}

(Received 10 November 1998)

\begin{abstract}
A supersymmetric way of imposing the constraint of no double occupancy in models with strong on-site Coulomb repulsion is presented in this paper. In this formulation the physical operators in the constrainted Hilbert space are invariant under local unitary transformations mixing boson and fermion representations. To illustrate, the formulation is applied to the Heisenberg, $t-J$, and infinite- $U$ Anderson models. In particular, we study the Heisenberg model carefully in two dimensions where results on spin excitation spectrum are presented. [S0163-1829(99)50210-5]
\end{abstract}

The $t$ - $J$ model has become a focus in the study of strongly correlated metals and high- $T_{c}$ superconductors since it was proposed in the late 1980s. ${ }^{1}$ Because of the lack of small parameters for expansion, analytical understandings of the model were largely dependent on mean-field theories which treat the constraint of no double occupancy only on average. The most successful mean-field approaches to the $t-J$ model seem to be based on either the slave-fermion mean-field theory (SFMFT), ${ }^{2}$ which is successful at very small doping when antiferromagnetic correlation is important, and the slave-boson mean-field theory (SBMFT), ${ }^{3}$ which is successful at larger values of doping when the system becomes superconducting. The only difference between the two approaches is that two different representations of spin and electron operators are used to impose the constraint of no double occupancy. More recently, the focus in the study of high- $T_{c}$ superconductors has turned to the underdoped and spin-glass regimes where it is believed that the subtle interplay between antiferromagnetism and superconductivity determines the properties of this crossover region. In particular, several symmetries are proposed ${ }^{4,5}$ to understand the competition between antiferromagnetism and superconductivity in this regime of high- $T_{c}$ cuprates.

To understand the complicated behavior in this regime of the $t$ - $J$ model, it seems that a unified approach which incorporates both the advantages of the SFMFT and the SBMFT is essential. In this paper, we shall show that it is possible in general to formulate models with constraint of no double occupancy in a way which incorporates the advantages of both slave-fermion and slave-boson representations. In this formulation the physical operators are supersymmetric and are invariant under unitary transformations mixing fermion and boson representations. The formulation suggests that supersymmetry exists naturally in strongly correlated systems where on-site Coulomb repulsions are strong. In the following, we shall consider several models to illustrate our approach. To begin, we first consider the Hilbert space of a lattice model with constraint of no double occupancy imposed.

The constraint of no double occupancy implies that there are three possible states on any single lattice site in the model. The site can be either empty (hole state), or can be occupied by either an up- or down-spin electron. In the slave-boson(fermion) approach, the hole state is represented as a boson (fermion), whereas spins are represented as fermions (bosons). ${ }^{2,3}$ In our formulation we shall consider an enlarged Hilbert space where both possibilities of representing hole and spins coexist as different states of the system, i.e., there are now six possible states per site in the Hilbert space, represented by,

$$
\left|\sigma_{i}^{(f)}\right\rangle=c_{i \sigma}^{+}|0\rangle, \quad\left|h_{i}^{(b)}\right\rangle=b_{i}^{+}|0\rangle
$$

and

$$
\left|\sigma_{i}^{(b)}\right\rangle=\bar{Z}_{i \sigma}|0\rangle, \quad\left|h_{i}^{(f)}\right\rangle=f_{i}^{+}|0\rangle,
$$

where $\sigma=\uparrow, \downarrow, c_{i \sigma}^{+}$and $\bar{Z}_{i \sigma}$ are fermionic and bosonic spin creation operators, respectively, and $|0\rangle$ is the vacuum state. Similarly, $b_{i}^{+}$and $f_{i}^{+}$are bosonic and fermionic hole creation operators, respectively. Notice that we have separated the states into "slave-boson", (1a) and "slave-fermion", (1b) groups in Eq. (1). For a system of $N$-lattice sites, both groups of states are allowed at all sites in our formulation and the total Hilbert space is thus $2^{N}$ times larger than the Hilbert space of the original model. The essence of our approach is to construct a Hamiltonian which is equivalent to the original model in all these $2^{N}$ groups of states, and consequently our system is equivalent to $2^{N}$ replicas of the original model. To see how this Hamiltonian can be constructed for the $S=1 / 2$ Heisenberg model we first consider spin operators.

We consider the spin operator $\vec{s}_{i}$ at site $i$,

$$
\vec{s}_{i}=\vec{s}_{i}^{(f)}+\vec{s}_{i}^{(b)},
$$

where

$$
\vec{s}_{i}^{(f(b))}=\left(c^{+}(\bar{Z})_{i \uparrow}, c^{+}(\bar{Z})_{i \downarrow}\right) \vec{\sigma}\left(\begin{array}{c}
c(Z)_{i \uparrow} \\
c(Z)_{i \downarrow}
\end{array}\right),
$$

and $\vec{\sigma}$ 's are the usual Pauli matrix. Notice that $\vec{s}^{(f)}$ and $\vec{s}^{(b)}$ are the spin operators in slave-boson and slave-fermion representations, respectively. It is obvious that $\vec{s}_{i}$ is itself a spin operator since it is the sum of two spin operators. The matrix elements $\left\langle\sigma_{i}^{(\alpha)}\left|\vec{s}_{i}\right| \sigma_{i}^{\prime(\beta)}\right\rangle$ where $\alpha, \beta=f, b$ and $\sigma, \sigma^{\prime}=\uparrow, \downarrow$ can be computed easily where it is easy to see that 
$\left\langle\sigma_{i}^{(f)}\left|\vec{s}_{i}\right|{\sigma^{\prime}}_{i}^{(f)}\right\rangle=\left\langle\sigma_{i}^{(b)}\left|\vec{s}_{i}\right|{\sigma_{i}^{\prime(b)}}_{i}\right\rangle$ and gives the usual spin operator matrix elements between spin-1/2 states whereas all other matrix elements with $\alpha \neq \beta$ are equal to zero. Thus the states $\left|\sigma_{i}^{(f)}\right\rangle$ and $\left|\sigma_{i}^{(b)}\right\rangle$ together form two identical replicas of spin-1/2 states on site $i$ with our definition of spin operator (2). It follows that the Hamiltonian

$$
H_{J}=J \sum_{\langle i, j\rangle} \vec{s}_{i} \cdot \vec{s}_{j},
$$

represents $2^{N}$ identical copies of Heisenberg interaction in our enlarged Hilbert space.

The electron annihilation and creation operators in our system are defined as $\psi_{i \sigma}=h_{i}^{+} \xi_{i \sigma}$, and $\psi_{i \sigma}^{+}=\xi_{i \sigma}^{+} h_{i}$, respectively, where

$$
h_{i}=\left(\begin{array}{l}
f_{i} \\
b_{i}
\end{array}\right), \quad \xi_{i \sigma}=\left(\begin{array}{c}
Z_{i \sigma} \\
c_{i \sigma}
\end{array}\right),
$$

are doublets of hole and spin operators. It is straightforward to show that the electron operators defined this way satisfy the usual electron commutation relations within the Hilbert space spanned by Eq. (1). The kinetic energy term $H_{t}$ of the $t-J$ model can be constructed by requiring that the hopping matrix elements $\left\langle\sigma_{j}^{\alpha^{\prime}}, h_{i}^{\beta^{\prime}}\left|H_{t}\right| h_{j}^{\alpha}, \sigma_{i}^{\beta}\right\rangle=-t_{i j} \delta_{\alpha \alpha^{\prime}} \delta_{\beta \beta^{\prime}}$, where $\alpha, \alpha^{\prime}, \beta, \beta^{\prime}=b, f$, and $\left|h_{j}^{\alpha}, \sigma_{i}^{\beta}\right\rangle$ represent a state with a hole belonging to group $\alpha$ on site $j$ and a spin $\sigma$ belonging to group $\beta$ on site $i$. Notice that the group indices $\alpha$, $\beta$ 's are "conserved" at each site in constructing $H_{t}$. It is straightforward to show that

$$
\begin{aligned}
H_{t}= & -t \sum_{\langle i, j\rangle, \sigma}\left(c_{j \sigma}^{+} b_{j} b_{i}^{+} c_{i \sigma}+c_{j \sigma}^{+} b_{j} f_{i}^{+} Z_{i \sigma}+\bar{Z}_{j \sigma} f_{j} b_{i}^{+} c_{i \sigma}\right. \\
& \left.-\bar{Z}_{j \sigma} f_{j} f_{i}^{+} Z_{i \sigma}+\text { c.c. }\right)
\end{aligned}
$$

where we have considered a Hamiltonian with nearest neighbor hopping only. The four different terms in $H_{t}$ place the matrix elements of the hopping term between the four possible combination of groups of states at sites $\langle i, j\rangle$. With this it follows that our system with Hamiltonian $H=H_{J}+H_{t}$ is equivalent to $2^{N}$ copies of the usual $t-J$ model. Notice that the hopping term cannot be simply represented as $H_{t}=$ $-t \Sigma\left(\psi_{i \sigma}^{+} \psi_{j \sigma}+\right.$ c.c. $)$ because of the sign difference in the "slave-fermion', term $\bar{Z}_{j \sigma} f_{j} f_{i}^{+} Z_{i \sigma}$. This sign difference is well known in studies of SFMFT. ${ }^{2}$

The invariance of our system under change of group of states defined in Eq. (1) at any lattice site can be expressed in the language of supersymmetry. To see that first we note that the spin operator (2) can be written using the $\xi_{\sigma}$ fields as

$$
s_{i}^{z}=\frac{1}{2}\left(\xi_{i \uparrow}^{+} \xi_{i \uparrow}-\xi_{i \downarrow}^{+} \xi_{i \downarrow}\right), \quad s_{i}^{+}=\xi_{i \uparrow}^{+} \xi_{i \downarrow}, \quad s_{i}^{-}=\xi_{i \downarrow}^{+} \xi_{i \uparrow},
$$

where $\xi_{i \sigma}^{+} \xi_{i \sigma^{\prime}}=\bar{Z}_{i \sigma} Z_{i \sigma^{\prime}}+c_{i \sigma}^{+} c_{i \sigma}$. It is now easy to see that the spin and electron operators are invariant under the superunitary transformation

$$
\xi_{i \sigma} \rightarrow U_{i} \xi_{i \sigma}, \quad h_{i} \rightarrow U_{i} h_{i}
$$

where $U_{i}$ 's are local $2 \times 2$ unitary super-matrices mixing the fermion and boson representations of spins and holes. The generators of the superunitary transformations are superspin operators $S_{i}^{z}, S_{i}^{x}=\left(S_{i}^{+}+S_{i}^{-}\right) / 2$ and $S_{i}^{y}=\left(S_{i}^{+}-S_{i}^{-}\right) /(2 i)$, where

$$
\begin{gathered}
S_{i}^{z}=\frac{1}{2}\left[\left(\sum_{\sigma} c_{i \sigma}^{+} c_{i \sigma}+b_{i}^{+} b_{i}\right)-\left(\sum_{\sigma} \bar{Z}_{i \sigma} Z_{i \sigma}+f_{i}^{+} f_{i}\right)\right], \\
S_{i}^{+}=\sum_{\sigma} c_{i \sigma}^{+} Z_{i \sigma}-b_{i}^{+} f_{i}, \\
S_{i}^{-}=\sum_{\sigma} \bar{Z}_{i \sigma} c_{i \sigma}-f_{i}^{+} b_{i},
\end{gathered}
$$

where we also define the (magnitude) ${ }^{2}$ of the superspin as $S^{2}=S^{z} S^{z}+\frac{1}{2}\left(S^{+} S^{-}+S^{-} S^{+}\right)$. Using the fact that the allowed states in our Hilbert space can only be singly occupied by either spin or hole, it is easy to show that $S_{i}^{2}$ $=\frac{3}{4}\left(\Sigma_{\sigma} \xi_{i \sigma}^{+} \xi_{i \sigma}+h_{i}^{+} h_{i}\right)=\frac{3}{4}$, where we have used the requirement that $\Sigma_{\sigma}\left(\bar{Z}_{i \sigma} Z_{i \sigma}+c_{i \sigma}^{+} c_{i \sigma}\right)+f_{i}^{+} f_{i}+b_{i}^{+} b_{i}=1$ in our Hilbert space (1) in deriving the equality. Notice that the constraint of no double occupancy implies that the magnitudes of the superspins are fixed $(=1 / 2)$ on all lattice sites. In terms of the superspin operators, the slave-boson and slavefermion representations are equivalent to fixing the direction of superspins to be pointing up and down, respectively, and supersymmetry expresses the fact that the physical observables in the system are, in fact, invariant under local (but time-independent) "rotations" in the superspin space. Notice that because of the "-, sign in the "slave-fermion" hopping term $\bar{Z}_{j \sigma} f_{j} f_{i}^{+} Z_{i \sigma}$, supersymmetry is straightly speaking, broken by the $t$ - term in the $t$ - $J$ model. However, our formulation of $t-J$ model in the enlarged Hilbert space (1) does not require supersymmetry in the Hamiltonian and is still valid.

The Lagrangian of the $t-J$ model in the enlarged Hilbert space is

$$
\begin{aligned}
L= & i \hbar \sum_{i, \sigma} \xi_{i \sigma}^{+} \frac{\partial}{\partial t} \xi_{i \sigma}+i \hbar \sum_{i} h_{i}^{+} \frac{\partial}{\partial t} h_{i}-H_{t}-H_{J} \\
& +\sum_{i} \lambda_{i}\left(\sum_{\sigma} \xi_{i \sigma}^{+} \xi_{i \sigma}+h_{i}^{+} h_{i}-1\right)-\mu \sum_{i} h_{i}^{+} h_{i},
\end{aligned}
$$

and a Path-integral formulation for the system can be written down as usual. In particular, the partition function of the present model is $2^{N}$ times the partition function of the original $t$ - $J$ model. Notice that the constraint of no double occupancy in our enlarged Hilbert space is imposed by a Lagrange multiplier term as usual.

It is obvious that the same approach can be applied to other models with constraint of no double occupancy, as long as a proper Hamiltonian can be constructed in the enlarged Hilbert space. For example, we find that the Hamiltonian for the infinite- $U$ Anderson model in the supersymmetric representation is ${ }^{6}$ 


$$
\begin{aligned}
H_{a n d}= & \sum_{\vec{k}, \sigma} \epsilon_{\vec{k}} a_{\vec{k} \sigma}^{+} a_{\vec{k} \sigma}+\epsilon_{o} \sum_{i, \sigma} \xi_{i \sigma}^{+} \xi_{i \sigma} \\
& +V \sum_{i, \sigma}\left(a_{i \sigma}^{+} \psi_{i \sigma}+\psi_{i \sigma}^{+} a_{i \sigma}\right),
\end{aligned}
$$

where $a\left(a^{+}\right) \vec{k}$ is the annihilation(creation) operator for conduction electrons with momentum $\vec{k}$ and spin $\sigma$ and $\xi\left(\xi^{+}\right)_{i \sigma}$ are annihilation(creation) operator for localized spins on site $i$ where constraint of no double occupancy is imposed. $\epsilon_{k}$, $\epsilon_{o}$, and $V$ have their usual meaning in Anderson model. The spin and electron operators in the localized orbitals are represented by $\xi_{i \sigma}$ and $\psi_{i \sigma}$ operators which are supersymmetric in our formulation and the constraint of no double occupancy can be imposed by Langrange multiplier fields as in the $t-J$ model. Notice that the Heisenberg and infinite- $U$ Anderson models are completely supersymmetric, in contrast to the $t-J$ model where supersymmetry is broken by the $t$ - term.

To illustrate further the present formulation we consider the $S=1 / 2$ Heisenberg model in two dimensions in more detail. Early theoretical analysis showed that the low energy physics of the model should be described by the renormalized classical regime of the $(2+1) d$ non-linear- $\sigma$ model $(\mathrm{NL} \sigma \mathrm{M}){ }^{7}$ However it was found recently that the length scale at which the $\sigma$-model description is valid is surprisingly long. ${ }^{8,9}$ Moreover the low-energy excitation spectrum of the model on a finite-size lattice indicates significant discrepancy compared with predictions of NL $\sigma \mathrm{M}$, even when the size of the system is rather large. ${ }^{10}$ We shall see how the supersymmetric formulation of the Heisenberg model offers a plausible solution to the problem.

Using the constraint $\Sigma_{\sigma} \xi_{i \sigma}^{+} \xi_{i \sigma}=1$, it is straightfoward to show that $\vec{s}_{i} \cdot \vec{s}_{j}$ can be written as

$$
\begin{aligned}
\vec{s}_{i} \cdot \vec{s}_{j}= & \frac{1}{4}+\frac{1}{2}\left(\xi_{i \uparrow}^{+} \xi_{i \downarrow} \xi_{j \downarrow}^{+} \xi_{j \uparrow}+\xi_{i \downarrow}^{+} \xi_{i \uparrow} \xi_{j \uparrow}^{+} \xi_{j \downarrow}\right. \\
& \left.-\xi_{i \uparrow}^{+} \xi_{i \uparrow} \xi_{j \downarrow}^{+} \xi_{j \downarrow}-\xi_{i \downarrow}^{+} \xi_{i \downarrow} \xi_{j \uparrow}^{+} \xi_{j \uparrow}\right) \\
= & \frac{1}{4}-\frac{1}{2} \sum_{\alpha, \beta} \Delta_{i j}^{\alpha \beta+} \Delta_{i j}^{\alpha \beta}
\end{aligned}
$$

where $\Delta_{i j}^{\alpha \beta}=\alpha_{i \uparrow} \beta_{j \downarrow}-\alpha_{i \downarrow} \beta_{j \uparrow}, \alpha_{\sigma}, \beta_{\sigma}=\left(Z_{\sigma}, c_{\sigma}\right)$ and $\Delta_{i j}^{\alpha \beta+}$ is the Hermitian conjugate of $\Delta_{i j}^{\alpha \beta}$. A mean-field theory can be obtained by making the decoupling $\Delta_{i j}^{+} \Delta_{i j} \rightarrow\left\langle\Delta_{i j}^{+}\right\rangle \Delta_{i j}$ $+\Delta_{i j}^{+}\left\langle\Delta_{i j}\right\rangle-\left\langle\Delta_{i j}^{+}\right\rangle\left\langle\Delta_{i j}\right\rangle,{ }^{11}$ with corresponding mean-field Hamiltonian

$$
\begin{aligned}
H_{m f}= & J \sum_{i, j}\left(\left\langle\Delta_{i j}^{+}\right\rangle \Delta_{i j}+\Delta_{i j}^{+}\left\langle\Delta_{i j}\right\rangle-\left\langle\Delta_{i j}^{+}\right\rangle\left\langle\Delta_{i j}\right\rangle\right) \\
& +\sum_{i} \lambda\left(\sum_{\sigma} \xi_{i \sigma}^{+} \xi_{i \sigma}\right)
\end{aligned}
$$

where $\langle A\rangle$ is the expectation value of operator $A$ evaluated with the mean-field Hamiltonian. The mean-field parameter $\lambda$ is determined by the condition $\Sigma_{\sigma}\left(\left\langle\bar{Z}_{i \sigma} Z_{i \sigma}\right\rangle+\left\langle c_{i \sigma}^{+} c_{i \sigma}\right\rangle\right)$ $=1$. Notice that both slave-boson and slave-fermion meanfield parameters are incorporated in the supersymmetric mean-field theory (SUMFT). We shall be interested in trans- lationally invariant solutions where the mean-field parameters are independent of $\langle i, j\rangle$ in the following.

Using the fact that $\langle C\rangle=0$ for Grassman variables $C$ 's, we find that $\left\langle\Delta_{i j}^{Z c}\right\rangle=\left\langle\Delta_{i j}^{c Z}\right\rangle=0$. However, both bosonic and fermionic terms $\left\langle\Delta_{i j}^{Z Z}\right\rangle$ and $\left\langle\Delta_{i j}^{c c}\right\rangle$ are still present in the mean-field Hamiltonian. The relative weight of the two mean-field terms are determined by minimizing the free energy of the system with the constraint that the average number of spins is equal to one on every site.

Solving the mean-field equations we find that there are in general two solutions corresponding to local minima in the mean-field free energy. The two solutions have either $\left\langle\Delta_{i j}^{Z Z}\right\rangle \neq 0$ and $\left\langle\Delta_{i j}^{c c}\right\rangle=0$ or the other way round and are generalizations of the usual slave-fermion and slave-boson solutions. Notice that supersymmetry is broken in these meanfield solutions. Comparing the free energies of the two solutions we find that the solution with $\left\langle\Delta_{i j}^{Z Z}\right\rangle \neq 0$ has lower free energy at all temperatures (at high temperature $T$ $>0.6 \mathrm{~J},\left\langle\Delta_{i j}^{Z Z}\right\rangle$ is also zero, corresponding to a hightemperature phase in the mean-field solution) corresponding to a generalization of usual Schwinger-boson mean-field theory (SCMFT) (Ref. 11) description of the Heisenberg model. Notice that SCMFT is qualitatively similar to NL $\sigma \mathrm{M}$ and offers a fairly accurate description of the lowtemperature properties of the Heisenberg model in two dimensions. $^{11}$

The main difference between the SUMFT and SCMFT is that although both mean-field solutions have only $\left\langle\Delta_{i j}^{Z Z}\right\rangle$ $\neq 0$, spin excitations with both boson and fermion statistics are present in SUMFT. Because of the constraint term, fermionic spin excitations appear as dispersionless high-energy excitations with energies $\lambda$ in the SUMFT, where $\lambda \sim 2.3 J$ numerically. Notice that the existence of fermion spin excitations in Heisenberg antiferromagnets has been demonstrated within the framework of SCMFT (Ref. 12) and resonant valence bond wave functions. ${ }^{13}$ The SUMFT provides a more quantitative framework to study these excitations. The appearance of fermionic spin excitations in SUMFT implies that at intermediate energy $\epsilon \sim J$, the behavior of the SUMFT

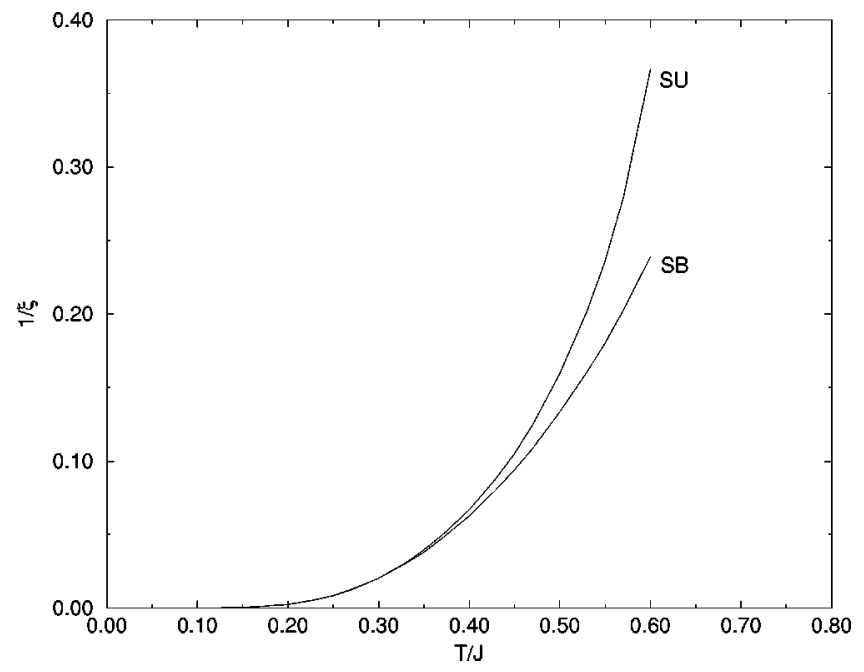

FIG. 1. Inverse antiferromagnetic spin-spin correlation length $\xi(T)^{-1}$ as a function of temperature: $\xi_{S C}^{-1}$, from SCMFT; $\xi_{S U}^{-1}$, from SUMFT. 
is quite different from that of NLoM or SCMFT, which describes only bosonic spinwave excitations. Besides a difference in the dynamics structure factor $S(\vec{q}, \omega),{ }^{14}$ the system described by SUMFT is more disordered at temperature $T$ $\neq 0$ because of the appearance of the extra degrees of freedom. In Fig. 1 we show the temperature dependence of the inverse antiferromagnetic spin correlation length $\xi(T)^{-1}$ as a function of temperature, for both the cases of SCMFT $\left(\left[\xi_{S C}(T)\right]^{-1}\right)$ and the new SUMFT $\left(\left[\xi_{S U}(T)\right]^{-1}\right)$. Notice that the correlation length is shorter in the supersymmetric theory at $T \neq 0$ and the difference is quite significant even when antiferromagnetic correlation is still strong $[\xi(T)$ $\sim 5-10$ lattice sites], in agreement with Monte-Carlo results. ${ }^{9}$

Our supersymmetric formulation can also be applied to study the $S=1 / 2$ Heisenberg model in one dimension. Assuming a mean-field solution which does not break supersymmetry and expanding around the mean-field solution, we obtain a supersymmetric gauge-field theory. In $(1+1) d$ the gauge fields impose constraints in the spin-field $\xi_{\sigma}(x)$ which can be solved exactly by bosonizing the $\xi_{\sigma}(x)$ field properly. ${ }^{15}$ The resulting bosonized Lagrangian describes spins as semions with correct spin-spin correlation function for the Heisenberg model in one dimension. ${ }^{15}$

Summarizing, we present in this paper a formulation for lattice models with constraints of no double occupancy where supersymmetry is inherent in the physical observables. The existence of this hidden supersymmetry is demonstrated explicitly in several models where supersymmetric representation of the models are presented. As an example, we apply our new formulation to the $S=1 / 2$ two-dimensional (2D) Heisenberg model where we find within mean-field theory that the system has long-ranged antiferromagnetic order with low-energy spinwave excitations at zero temperature. However, fermionic spin excitations which are absent in the NL $\sigma \mathrm{M}$ description exist at intermediate energies $\epsilon$ $\sim J$. The existence of fermionic excitations may offer an explanation for the recently discovered anomalous behaviors in two-dimensional Heisenberg antiferromagnets. We also find that our supersymmetric formulation produces the correct known results of 1D Heisenberg model.

We acknowledge the support of HKRGC through Grant No. HKUST6143/97P.
${ }^{1}$ P. W. Anderson, Science 235, 1196 (1987).

${ }^{2}$ C. Jayaprakash, H. R. Krishnamurthy, and S. Sarker, Phys. Rev. B 40, 2610 (1989); C. L. Kane, P. A. Lee, T. K. Ng, B. Chakraborty, and N. Read, ibid. 41, 2653 (1990).

${ }^{3}$ G. Baskaran, Z. Zou, and P. W. Anderson, Solid State Commun. 63, 973 (1987); C. Gros, R. Joynt, and T. M. Rice, Phys. Rev. B 36, 381 (1987); G. Kotliar and J. Liu, ibid. 38, 5142 (1988).

${ }^{4}$ X.-G. Wen and P. A. Lee, Phys. Rev. Lett. 76, 503 (1996)

${ }^{5}$ S. C. Zhang, Science 275, 1089 (1997).

${ }^{6}$ see C. Pepin and M. Lavagna, Z. Phys. B 103, 259 (1997) for a similar formulation.

${ }^{7}$ S. Chakravarty, B. I. Halperin, and D. R. Nelson, Phys. Rev. B 39, 2344 (1989); see also P. Hasenfratz and F. Niedermayer, Phys. Lett. B 269, 231 (1991).
${ }^{8}$ M. Greven et al., Phys. Rev. Lett. 72, 1096 (1994).

${ }^{9}$ B. B. Beard et al., Phys. Rev. Lett. 80, 1742 (1998); J-K. Kim and M. Troyer, ibid. 80, 2705 (1998).

${ }^{10}$ C. Lavalle et al., Phys. Rev. Lett. 80, 1746 (1998).

${ }^{11}$ D. P. Arovas and A. Auerbach, Phys. Rev. B 38, 316 (1988); see also D. Yoshioka, J. Phys. Soc. Jpn. 58, 1516 (1989).

${ }^{12}$ T. K. Ng, Phys. Rev. B 52, 9491 (1995).

${ }^{13}$ N. Read and B. Chakraborty, Phys. Rev. B 40, 7133 (1989).

${ }^{14}$ Notice that the fermionic spin excitations will have finite dispersion when treated beyond mean-field theory.

${ }^{15}$ P. A. Marchetti, Z-B. Su, and L. Yu, Nucl. Phys. B: Field Theory Stat. Syst. 482 [FS], 731 (1996); F. D. M. Haldane, in Electron Correlation in Narrow Band Systems, edited by T. Moriya (Springer, Berlin, 1981). 